\title{
Un modelo para la música: teoría del ritmo*
}

A Model for Music: Theory of Rhythm

Jesús Lara Popoca ${ }^{\dagger}$

\section{Resumen}

En este artículo me propongo utilizar métodos conjuntistas para elaborar la teoría del ritmo musical. Partiendo de una noción de estructura musical rigurosamente definida, el trabajo desarrolla los conceptos básicos de la teoría del ritmo extendiendo los conceptos presentados en Lara (inédito) para poder plantear posteriormente un modelo para la teoría musical que comprenda tanto la teoría de las alturas como la del ritmo.

Palabras clave: métodos conjuntistas - teoría del ritmo musical - teoría de las alturas del sonido - computabilidad y decidibilidad de teorías musicales

\begin{abstract}
My aim in this paper is to use set-theoretical methods in order to elaborate a theory of musical rhythm. Departing from a rigorously defined notion of musical structure, it develops the basic concepts of the theory of rhythm, extending the concepts presented in Lara (unpublished) in order to propose a model for musical theory that embraces both the theory of pitches and that of rhythm.
\end{abstract}

Keywords: set-theoretical methods - theory of musical rhythm - theory of sound pitch - computability and decidability of musical theories

\footnotetext{
* Recibido: 27 de marzo de 2013. Aceptado con revisiones: 18 de julio de 2013.

+ Universidad Veracruzana (UV), México. Para contactar al autor, por favor, escribir a: jesuslara_77@yahoo.com. Metatheoria 6(1)(2015): 73-78. ISSN 1853-2322. eISSN 1853-2330.

(c) Editorial de la Universidad Nacional de Tres de Febrero. Publicado en la República Argentina.
} 
El presente trabajo tiene como finalidad desarrollar los conceptos básicos de la teoría del ritmo, extender los conceptos presentados en Lara (inédito) para, y así, poder plantear posteriormente un modelo para la teoría musical que comprenda tanto la teoría de las alturas como la del ritmo. Como punto de partida se define un posible universo en el que se pueda modelar el comportamiento de los objetos sonoros a través del tiempo; posteriormente, se definen los conceptos fundamentales de la teoría del ritmo de la misma manera que se hizo para la teoría de las alturas.

En la teoría de las alturas se estudia la relación atemporal entre distintos objetos del conjunto de frecuencias $N$, mientras que en la teoría del ritmo se estudia la interacción en el tiempo de estos elementos, con lo que surgen nuevos objetos de estudio, a saber, objetos o relaciones temporales de miembros o subconjuntos de $N$. Para integrar ambas teorías es necesario comprender la forma en que se relacionan de manera atemporal los elementos de $\mathrm{N}$, y como se enriquece nuestro estudio de esas relaciones al considerar el factor temporal. Es importante notar que las relaciones que se definen en Lara (inédito) siguen siendo válidas en este nuevo contexto, solo que ahora debemos considerar, por ejemplo, la relación atemporal de dos sonidos de acuerdo con lo que se presenta en Lara (inédito), y después considerar el contexto temporal, es decir, si los sonidos son simultáneos o no, y de qué manera afecta el que lo sean o no al efecto de la composición.

De manera intuitiva podemos observar que en esta nueva etapa de desarrollo el análisis se vuelve en cierto sentido bidimensional, pues por un lado se estudia la relación entre elementos del conjunto de frecuencias posibles (alturas) y por otro se estudia la relación entre los instantes de tiempo en que suceden. El análisis musical ocurre al considerar la función de un objeto (altura o conjunto de alturas) dependiendo de su posición en el tiempo. En los lenguajes naturales sucede algo parecido, por ejemplo con la palabra "instrumento". Si consideramos los dos enunciados "El instrumento produce un sonido hermoso" y "El hombre toca el instrumento" vemos que la misma palabra tiene funciones diferentes, aunque en este caso la función no depende del tiempo si no de la posición o el lugar que ocupa la palabra en el enunciado. En la música sucede algo parecido, pues el mismo objeto tiene diferentes funciones dependiendo del lugar en el tiempo que ocupe y del contexto sonoro que lo acompaña. ${ }^{1}$ Un objeto puede ser sintácticamente correcto de acuerdo con la teoría de las alturas, pero puede no serlo en el contexto de una composición por el lugar en que aparece. Esto depende desde luego de las convenciones propias a cada periodo y estilo musical.

Iniciamos la presente exposición con la definición de una estructura musical en la cual se pueden modelar las relaciones atemporales entre alturas musicales tal como aparece en Lara (inédito).

\section{Definición 1:}

$\mathfrak{M}$ es una estructura musical syss existen $N, \prec, K$ tales que

(0) $\mathfrak{M}=\langle N, \prec, k\rangle$

(1) $N$ es un conjunto no vacío

(2) $\prec$ es un orden total sobre $N$

(3) $k \in N$

(4) $\forall x \exists y(x \prec y \wedge \forall z(x \prec z \Rightarrow z=y \vee y \prec z)$

(5) $\forall x \exists y(y \prec x \wedge \forall z(z \prec x \Rightarrow z=y \vee z \prec y)$

El ritmo en la música es el elemento fundamental o primitivo en el sentido de que todo evento musical implica un evento rítmico. Es posible tener eventos musicales puramente rítmicos, mientras que no es posible tener eventos musicales de naturaleza puramente melódica o armónica. El ritmo está

\footnotetext{
${ }^{1}$ Un mismo acorde tiene funciones diferentes en tonalidades diferentes y dentro de una misma tonalidad puede funcionar como acorde definitivo o como acorde de paso, dependiendo del instante temporal en el que ocurra.
} 
íntimamente ligado a la existencia misma del fenómeno musical, pues un sonido que se prolonga de manera indefinida difícilmente puede ser catalogado como música. Es indispensable, mediante el uso del silencio, interrumpir la prolongación indefinida de este sonido, dando lugar de esta forma a la creación de un patrón rítmico ${ }^{2}$ y, por lo tanto, musical. Asimismo, el ritmo es importante para el fenómeno musical, pues introduce un orden en el tiempo al proporcionar un sistema de referencia con el cual a cada altura o conjunto de alturas se le asigna un lugar específico en el tiempo.

Procedamos pues con la construcción del conjunto sobre el que estaremos trabajando. El camino a seguir es formar el producto directo de una familia $\left\{\mathfrak{M}_{i}\right\}$ de estructuras musicales, en donde el conjunto de índices es un intervalo cerrado de números reales. La idea es contar con un conjunto en el que sea posible modelar los conceptos que se presentan en Lara (inédito) y desarrollar la maquinaria teórica para incorporar el ritmo.

\section{Definición 2:}

Sea $I \neq \varnothing$ un intervalo cerrado y, para cada $i \in I$, sea $\mathfrak{M}_{i}$ una estructura musical. Definimos el espacio musical como el conjunto

$$
\mathfrak{M}(I)=\left\{f: I \rightarrow \bigcup_{i \in I} N_{i}: f(i) \in N_{i} \quad \text { para cada } i \in I\right\}
$$

En la definición 2 el conjunto $I=[a, b]$ se elige de tal forma que el instante inicial de la pieza musical sea $a$ y el instante final igual a $b$.

Parte de la discusión desarrollada en el párrafo que sigue a la definición 1 queda formalizada en la siguiente

\section{Definición 3:}

Una melodía es un elemento de $\mathfrak{M}(I)$ que no es constante.

La coexistencia de dos o más melodías es lo que en un sentido general ${ }^{3}$ se puede definir como contrapunto.

\section{Definición 4:}

Dados dos elementos $f, g \in \mathfrak{M}(I)$ diremos que un evento $f\left(x_{0}\right)$ antecede a un evento $g\left(y_{0}\right)$ si $x_{0}<y_{0}$. Dos eventos $f\left(x_{0}\right), g\left(y_{0}\right)$ son simultáneos si $x_{0}=y_{0}$.

Observemos que si en la definición 4 tomamos $f=g$, esto implica que para $x_{0}<y_{0}$ se tiene que el evento $f\left(x_{0}\right)$ antecede al evento $f\left(y_{0}\right)$. La definición 4 es para la teoría del ritmo el equivalente al punto dos de la definición 1 para la teoría de las alturas. Ahora contamos con una forma de ordenar cada objeto de acuerdo a su altura (frecuencia) y a su posición en el tiempo. Esta relación entre altura y tiempo nos proporciona la herramienta fundamental para el planteamiento de un modelo para la música en el que sea posible formalizar o plantear algún tipo de herramienta de análisis musical.

\section{Definición 5:}

Un acorde es un conjunto de proyecciones de notas en el eje de las frecuencias.

\footnotetext{
2 Aun si el sonido no vuelve a producirse, el tiempo de duración es susceptible de ser interpretado como un evento rítmico. A partir de diferentes duraciones se pueden formar composiciones musicales puramente rítmicas.

${ }^{3}$ En un sentido estricto, el contrapunto es la coexistencia de dos o más melodías que obedecen un conjunto de reglas definidas que dependen principalmente del periodo musical en cuestión. El concepto de contrapunto de un periodo histórico puede ser muy diferente al de otro periodo, teniendo como elemento en común la existencia simultánea de dos o más melodías.
} 
En Lara (inédito) se definió una escala como un subconjunto $\xi$ del universo $N$ tal que $\operatorname{Card}(\xi) \geq 2$. Un acorde, en estricto sentido, es también un subconjunto de $N$, aunque por lo regular se considera que es de naturaleza diferente a la de una escala. La definición de escala no depende del tiempo, o de manera más precisa, no depende de un instante de tiempo en particular. Un acorde por lo regular se entiende como un conjunto de notas o alturas simultáneas. Lo complicado en el concepto tradicional de acorde es que la simultaneidad en cuestión no necesariamente se refiere a la simultaneidad que se maneja en la definición 4, Sino, más bien, a una especie de cercanía perceptual (enfatizada por la cercanía temporal de los eventos) en la que un conjunto de sonidos recibe el nombre de acorde. Un conjunto de eventos sonoros simultáneos (en el sentido de la definición 4) forman un acorde, pero un conjunto de eventos no simultáneos pueden también formar un acorde siempre y cuando su aparición en la obra musical cumpla con ciertos requisitos, a saber, que los intervalos formados entre los elementos del conjunto se ajusten a algún patrón predefinido y que los sonidos sean producidos de manera consecutiva. ${ }^{4}$ La generalidad en la presente definición tiene como finalidad incluir casos extremos en los que los elementos en cuestión pueden estar temporalmente alejados, pero que, al momento de analizar la pieza, conviene interpretarlos como miembros de un acorde.

Se ha mencionado de manera informal uno de los problemas fundamentales en el análisis musical, a la vez que se ha propuesto un medio para resolverlo, a saber, el definir de manera efectiva lo que es un acorde y cómo se puede estudiar o interpretar. La importancia de este problema radica en el siguiente hecho: dependiendo del tipo de pieza que se esté estudiando, los acordes, y, en consecuencia, la armonía de la pieza, pueden presentarse de manera implícita, esto es, que después de realizar un análisis de la pieza, la estructura, y, por tanto, el movimiento armónico de la pieza, puede ser diferente al que en un principio o puede aparentar ser diferente de manera inmediata.

De esta forma, la definición 5 incluye los casos posibles para un acorde (sonidos simultáneos o no) y establece una clara diferencia entre el concepto de escala y el de acorde.

Una escala es un subconjunto de $N_{i}$ para cada $i \in I$, mientras que el de acorde es un conjunto de proyecciones de elementos de $\mathfrak{M}(I)$ en un instante dado o en varios. Con esto se observa la diferencia entre un acorde como elemento sintáctico o semántico. Esta distinción cobra importancia al considerar por ejemplo acordes incompletos o inversiones. ${ }^{5} \mathrm{Al}$ realizar el análisis de una pieza, con frecuencia encontraremos un conjunto de notas que no se ajusta totalmente a la definición de un cierto tipo de acorde, pero que cumple con esa función dentro del contexto de la pieza. Digamos que el conjunto no es sintácticamente un acorde mayor, pero semánticamente sí lo es.

A pesar del aparente éxito ${ }^{6}$ de la definición 2 , el conjunto $\mathfrak{M}(I)$ resulta ser muy grande y complejo para la mayoría de las aplicaciones. La generalidad del espacio $\mathfrak{M}(I)$ permite modelar composiciones de una complejidad mayor a la que comúnmente se presenta en el repertorio occidental y en la música folklórica del mundo. ${ }^{7}$ Esto, aunque desde luego es una ventaja si se considera que uno de los principales requisitos que debe cumplir un modelo para la música es su generalidad, en la práctica complica considerablemente el manejo y la definición de los objetos musicales, por lo que resulta conveniente trabajar con un subconjunto de $\mathfrak{M}(I)$ de naturaleza menos compleja pero apropiado para nuestros fines.

\section{Definición 6:}

El espacio $\mathfrak{M}_{c}(I)$ es la restricción del espacio $\mathfrak{M}(I)$ al conjunto de las funciones escalonadas.

De manera intuitiva podemos ver que este conjunto es un candidato adecuado para servir como universo para un modelo de la teoría musical, ya que una nota se sostiene durante un periodo de

\footnotetext{
${ }^{4}$ Esto en la teoría musical se conoce como arpegio y no debe ser confundido con el concepto de melodía. Un arpegio no es un elemento de $\mathfrak{M}(I)$, sino un conjunto de proyecciones de uno o varios elementos de $\mathfrak{M}(I)$ en uno o varios instantes cercanos.

${ }^{5}$ El concepto de inversión no se desarrollara en el presente trabajo.

${ }^{6}$ A saber, posibilidad de definir de manera simple los conceptos de melodía y acorde.

${ }^{7}$ El conjunto $\mathfrak{M}(I)$ resulta apropiado para modelar el comportamiento de obras electro-acústicas.
} 
tiempo determinado antes de cambiar a una nueva altura. Durante este periodo por lo regular se considera que la frecuencia de la nota es constante.

Hemos llegado, pues, al momento culminante en esta exposición. Daremos a continuación las definiciones fundamentales en la teoría del ritmo. Para ello consideraremos un caso parecido al de la definición 5. Si, en lugar de considerar las proyecciones de elementos de $\mathfrak{M}(I)$ o $\mathfrak{M}_{c}(I)$ sobre el eje de las alturas, observamos las proyecciones sobre el conjunto de índices, entonces tendremos un subconjunto de puntos de I que estarán relacionados entre sí de manera independiente a la altura(frecuencia) que asuman los elementos de $\mathfrak{M}(I)$ en esos puntos.

Para motivar la definición de los conceptos rítmicos, consideremos el siguiente ejemplo: supongamos un elemento $f(t) \in \mathfrak{M}_{c}(I)$ definido de la siguiente forma

$$
f(t)= \begin{cases}\alpha_{1} & t_{0} \leq t \leq t_{1} \\ \alpha_{2} & t_{1} \leq t \leq t_{2} \\ \alpha_{3} & t_{2} \leq t \leq t_{3} \\ \alpha_{4} & t_{3} \leq t \leq t_{4}\end{cases}
$$

Considerando las proyecciones sobre el eje de las alturas, se pueden formar subconjuntos (acordes) tales como $\left\{\alpha_{1}, \alpha_{2}\right\},\left\{\alpha_{1}, \alpha_{4}\right\},\left\{\alpha_{3}, \alpha_{1}, \alpha_{4}\right\}$ y estudiar la relación que hay entre ellos de acuerdo con lo expuesto en Lara (inédito) y a las consecuencias que de ahí se siguen. Podemos también considerar el conjunto de proyecciones sobre $I$ que consta de los puntos en donde el elemento $f$ cambia de altura. De esta forma obtenemos un subconjunto $\left\{t_{0}, t_{1}, t_{2}, t_{3}, t_{4}\right\}$ de $I$. Ahora nos preguntamos, por ejemplo, qué relación hay entre las siguientes diferencias $t_{i}-t_{i-1}$ o del cociente $\frac{t_{i}-t_{i-1}}{t_{0}-t_{4}}$ para $i=\{1,2,3,4\}$. Estas y otras relaciones parecidas serán el objeto de estudio de la teoría del ritmo.

La teoría del ritmo, en términos generales, se puede ver como el estudio de las implicaciones de la siguiente

\section{Definición 7:}

Sea I un intervalo, definimos:

i) Pulso: Una partición regular $P_{0}$ de $I=[a, b]$.

ii) Compás: Dados $x_{i}, x_{j} \in P_{0}$ tales que $x_{i}<x_{j}$, un compás musical $C_{i}$ es un intervalo semiabierto $\left[x_{i}, x_{j}\right)$.

iii) Métrica: Dados un pulso $P_{0}$ y un compás $C_{i}$, definimos la métrica como $\operatorname{Card}\left(P_{0} \cap C_{i}\right)$.

iv) Dados $P_{0}, x_{k}, x_{k-1} \in P_{0}$ y $\Delta x_{k}=x_{k}-x_{k-1}$. Diremos que $\frac{\Delta x_{k}}{n}$ es una $n$ división de $\Delta x$. Dados $y_{k_{n}}$, $y_{k_{n+1}}$ elementos consecutivos de $\Delta x_{k},\left[y_{k_{n}}, y_{k_{n+1}}\right)$ es un n-eavo.

El pulso es el marco de referencia básico, el latido fundamental de la música. La función del metrónomo es indicar el pulso. ${ }^{8}$ El compás es la forma básica de organizar el discurso musical. Cada inicio de compás es un posible punto de partida o de llegada. ${ }^{9}$

La métrica es la duración o el tamaño del compás en términos del pulso. Por último, el punto (iv) de la definición 7 es la subdivisión del pulso. Gracias a esto se puede tener variedad rítmica.

A continuación se mencionan algunas consecuencias inmediatas, pero no del todo obvias, de los cuatro puntos de la definición 7 .

\footnotetext{
8 Esto significa que cada golpe o sonido del metrónomo está identificado con un punto único de la partición $P_{0}$.

9 Dependiendo del género y del país de origen, una composición puede estar estructurada con un diferente número de compases por sección, permitiendo así organizar el inicio de frases musicales (melódicas, armónicas, etc.) por número de compases.
} 
Sean $P_{0}, P_{1}$ dos pulsos. Si consideramos ambos pulsos como marcos de referencia simultáneos, tenemos entonces el concepto de polirritmia. Consideremos ahora una colección de compases $\left\{\mathrm{C}_{1}, \ldots\right.$, $\left.\mathrm{C}_{n}\right\}$ tales que la longitud de $\mathrm{C}_{i}$ es distinta a la longitud de $\mathrm{C}_{j}$ para $i \neq j$ y sea $P_{0}$ un pulso, luego

$$
\operatorname{Card}\left(P_{0} \cap C_{i}\right) \neq \operatorname{Card}\left(P_{0} \cap C_{j}\right) .
$$

Con esto se tienen métricas diferentes en una misma pieza. El estilo musical depende en gran medida del punto iv) de la definición 7.

Con el presente trabajo se logra un avance importante hacia la construcción de un modelo completo para la música que integre tanto la teoría de las alturas como la del ritmo. Queda todavía por mucho hacer. Entre otras cosas, desarrollar las implicaciones de las definiciones aquí presentadas y en Lara (inédito) para completar así el desarrollo teórico de la música dentro de este contexto formal; estudiar la posible incorporación de los conceptos presentados por Joseph Schillinger (1946) para enriquecer el presente desarrollo, en particular, explorar la posibilidad de incluir conceptos presentes en Schillinger (1946) como reglas de inferencia en nuestro sistema, abriendo con esto toda un nuevo campo de estudio de la teoría musical, por ejemplo, el estudio de la computabilidad y decidibilidad de teorías particulares, ${ }^{10}$ planteadas en el contexto del presente modelo de la música. Otro pendiente es, desde luego, el desarrollo del algoritmo capaz de analizar una pieza musical.

\section{Bibliografía}

Chang, C. C. y H. J. Keisler (1998), Model Theory, Amsterdam: North Holland.

Lara, J. (inédito), "Un modelo para la música: teoría de las alturas", manuscrito inédito.

Lateef, Y. A. (1981), Repository of Scales and Melodic Patterns, Amherst, MA: Fana Music.

Schenker, H. (1935), Neue musikalische Theorien und Phantasien, Vol. III: Der freie Satz, Vienna: Universal Editions.

Schillinger, J. (1946), The Schillinger System of Musical Composition, Vols. I and II, New York: Carl Fischer.

Slonimsky, N. (1947), Thesaurus of Scales and Melodic Patterns, New York: Charles Scribner's Sons.

\footnotetext{
${ }^{10}$ Como pueden ser, por ejemplo, la teoría del Jazz o la teoría dodecafónica.
} 